\title{
Study of HH production at CMS
}

\author{
Alexandra Oliveira ${ }^{* \dagger}$ \\ Dipartimento di Fisica e Astronomia and INFN, Sezione di Padova, Via Marzolo 8, I-35131 \\ Padova, Italy
}

CERN, 1211 Geneva 23, Switzerland

E-mail: alexandra.oliveira@cern.ch

\begin{abstract}
The production of the pairs of higgs bosons provides a direct handle on the structure of the Higgs field potential. While the di-higgs bosons production within the SM is very small and essentially out of the experimental reach within the Run I or II, several beyond SM theories foresee an enhancement that can be already probed with the available data. First searches for an inclusively produced heavy resonance that decays in pairs of higgs bosons within BSM theories, made using data collected during Run I by the CMS collaboration, are presented.
\end{abstract}

The European Physical Society Conference on High Energy Physics 22-29 July 2015

Vienna, Austria

${ }^{*}$ Speaker.
† On behalf of CMS collaboration 


\section{Interplay of different channels}

In general BSM new physics is supposed to couple to Higgs sector, consequently in many SM extensions exotic heavy resonances have the higgs and weak bosons among its main decays. The collider search for a heavy spin 0 or 2 resonance decaying to a pair of higgses benefists from the now well known higgs mass and the characterization of its decays [1,2].

A SM higgs boson with mass $125 \mathrm{GeV}$ decays preferentially to a b-quark pair (57.8\%), what seems to be confirmed by data. Therefore when searching for a pair of higgs bosons to restrict at least one of the bosons to decay to a $b \bar{b}$ pair guarantees a reasonable signal strength in comparison with other channels. This contribution is based in the Run I CMS searches for resonances decaying in pairs of higgs bosons in the $\gamma \gamma b \bar{b}, \tau \tau b \bar{b}$ and $b \bar{b} b \bar{b}$ channels $[3,4,5]$. If the higgs boson interacts with matter as predicted by SM the different searches we present represent respectively $0.26 \%$, $6.7 \%$ and $33 \%$ of $\mathrm{HH}$ branching fraction.

Figure (1) shows the observed and expected limits when the Standard Mode (SM) branching fractions are assumed to the Higgs boson and the heavy resonance is assumed as scalar. Those limits are overlaid with theory predictions for a spin- 0 particle so-called radion, commonly present of Warped Extra Dimensional class of models (see for example [6]). No significant excess was found in $8 \mathrm{TeV}$ data. In the following sections we detail a bit the different analyses. The specific $m_{X}$ value that defines this inflection point between channels depends on possible branching fractions variations for the $125 \mathrm{GeV}$ higgs boson (what can happen in specific models, for example with an enlarged Higgs sector of invisible higgs boson decays).

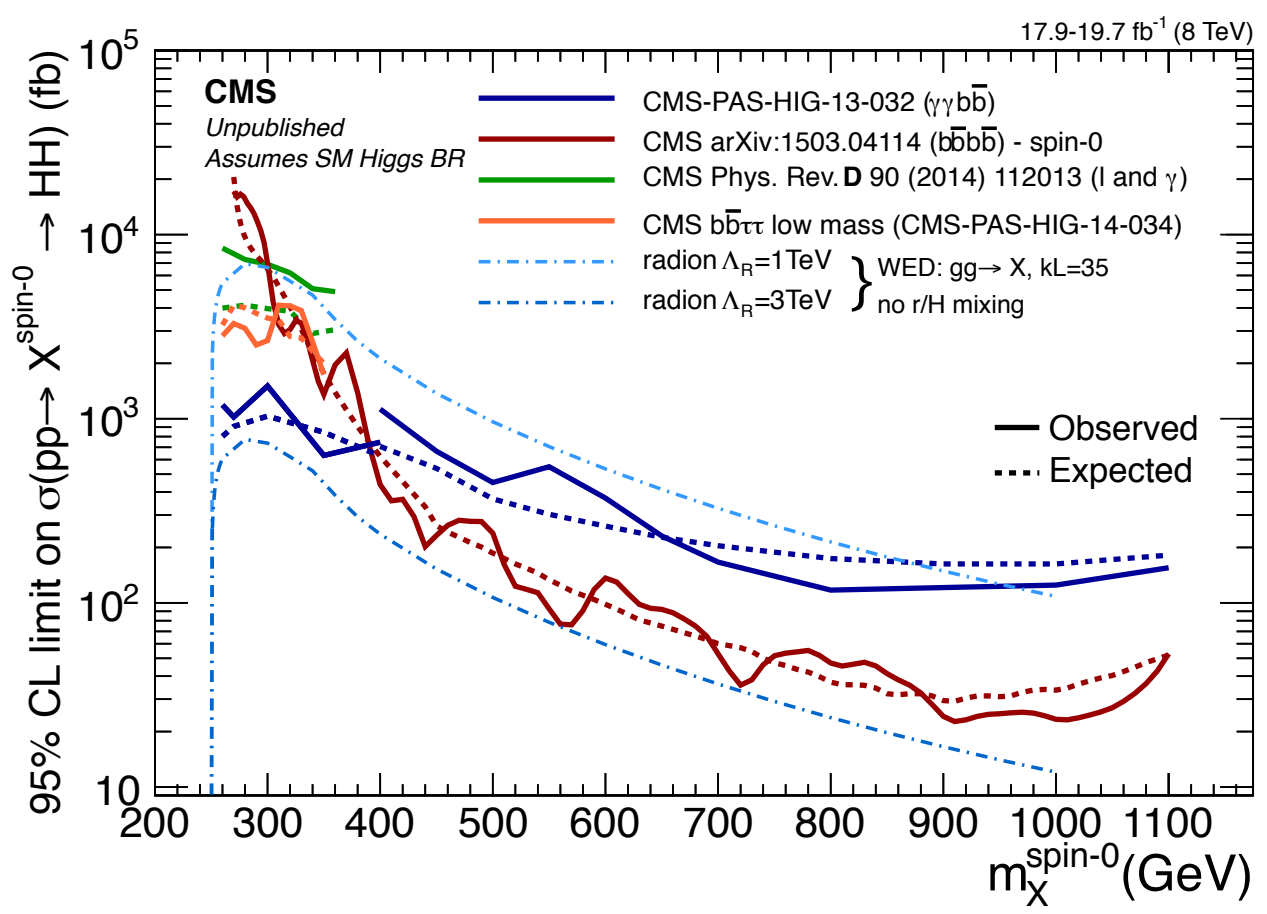

Figure 1: Resume of the CMS $8 \mathrm{TeV}$ results in the di-higgs channel in expected and observed limits. 


\section{The $\gamma \gamma b \bar{b}$ channel}

The data is triggered by the presence of two fotons. The analysis is based in the $\operatorname{SM} h \rightarrow \gamma \gamma$ search [7]. In this final state we have two fully reconstructed higgs boson. Up to jet multiplicity those are uniquely identified. Figure (2) shows the three invariant mass spectra available to exploit, after pre-selection of two fotons plus two jets. At this stage only at least one loose $b$-tagged jet is required.

The events was categorized as High Purity (HP) and Low Purity (LP) according with the number of medium $b$-tagged jets. The HP category corresponds to two medium $b$-tagged jets, while the LP to one medium $b$-tagged jet.
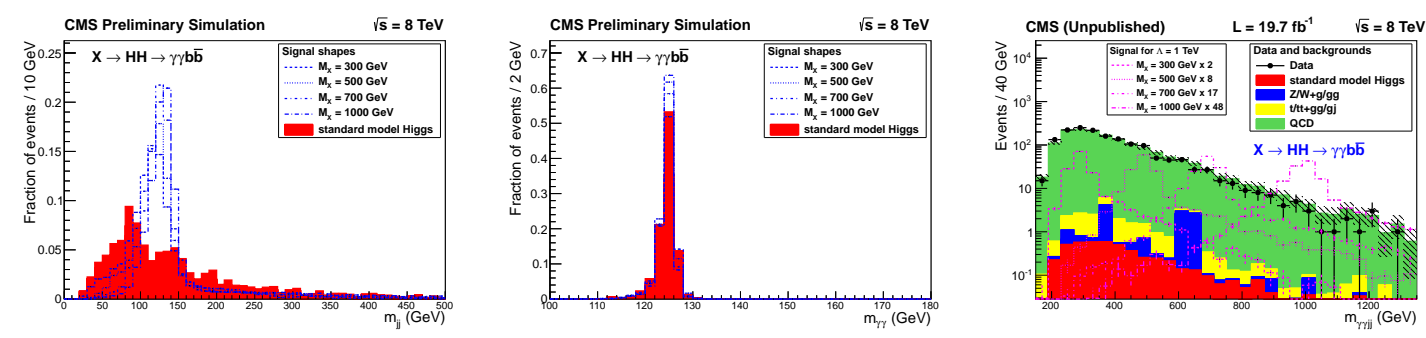

Figure 2: Invariant mass distributions used for signal characterization in the $\gamma \gamma b \bar{b}$ channel after selection of two fotons and requirement of two jets with at least one loose $b$-tag. In the single higgs spectra we show both resonant signal and its contamination by the SM-like single higgs boson production. In the four body mass we also show the main backgrounds.

Two different analyses were done, accordingly with the resonance mass hypothesis:

- low mass strategy $\left(m_{X}<\mathbf{4 0 0} \mathbf{~ G e V}\right)$ : The strategy is to select in 4 body and di-jet $\left(m_{j j}\right)$ invariant masses and fit a falling function in the $m_{\gamma \gamma}$ spectrum, taking advantage of the good signal resolution and controllable background shape. Figure (3) shows data and fitted background for low mass search after the selection corresponding to $m_{X}=300 \mathrm{GeV}$. The LP category is $\mathrm{O}(10)$ times more BKG populated, but contains half of the signal, helping to control the statistical uncertainty. The sensibility is however driven by the low statistic HP category.

- high mass strategy $\left(m_{X}>\mathbf{4 0 0} \mathbf{~ G e V}\right)$ : To select in four body mass to $m_{X}>400 \mathrm{GeV}$ reduces considerably the data statistics. To be able to have a reliable data-driven background estimation the analysis strategy is changed to select in and $m_{j j}$ and $m_{\gamma \gamma}$ mass spectra and fit a falling function in 4 body mass spectra. Figure (4) shows data and fitted background for low mass search after the high mass selection strategy.

The $8 \mathrm{TeV}$ analysis is dominated by statistical error. After the analysis selections of the low mass strategy the rate of SM single higgs production is reduced to less than $0.15(0.5)$ in the HP (LP) category. The signal deficiencies in function of the resonance mass are shown in figure (5). For signal hypotheses with $m_{X}>1 \mathrm{TeV}$ the signal efficiency start to drop due the fact the two photons that compose the triggered higgs start to merge in a jet. 

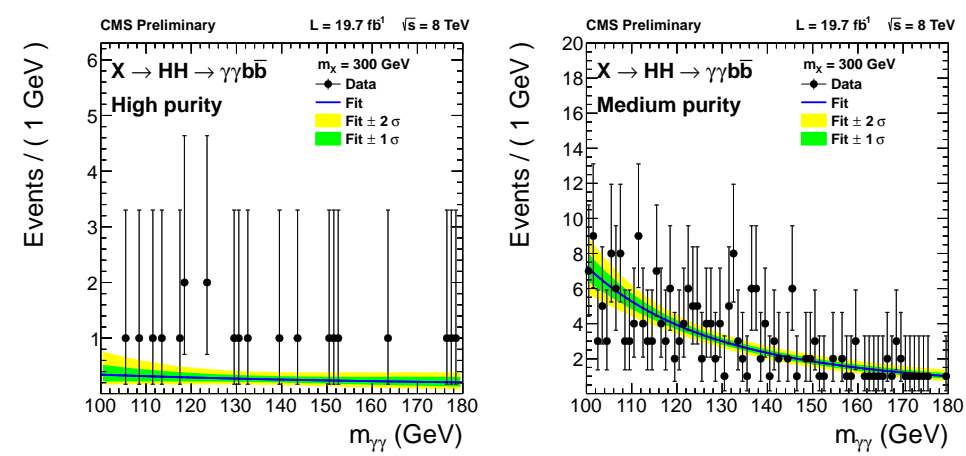

Figure 3: Events in $m_{\gamma \gamma}$ spectrum in medium-purity (left) and high-purity (right) categories for a low mass selection hypothesizing $m_{X}=300 \mathrm{GeV}$. The non-resonant component of the background is shown (black line) with 1 and $2 \sigma$ bands on the background estimation.
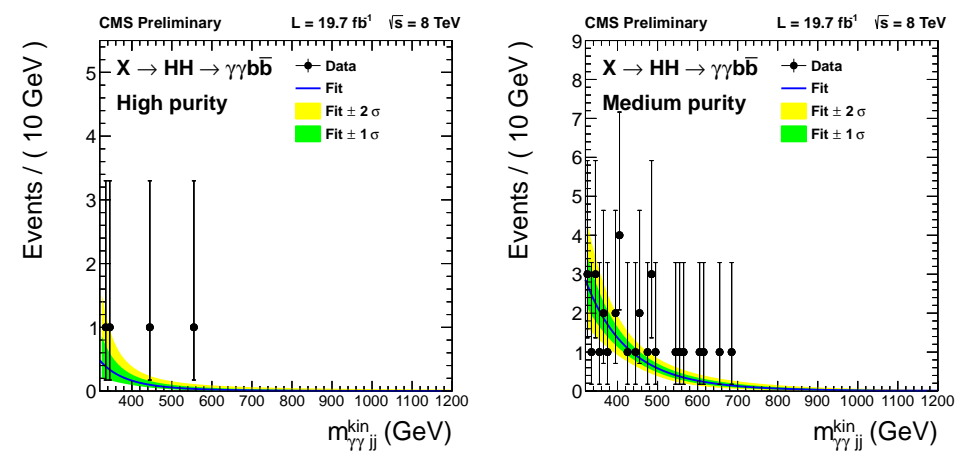

Figure 4: Events in $m_{\gamma \gamma j j}$ spectrum in medium-purity (left) and high-purity (right) categories. The nonresonant component of the background is shown (black line) with 1 and $2 \sigma$ bands on the background estimation.

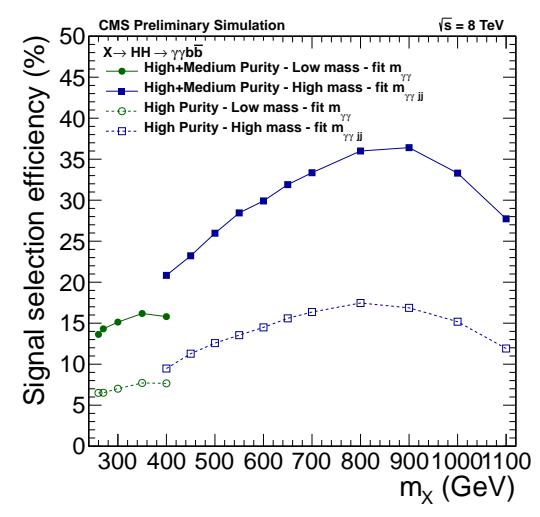

Figure 5: Signal efficiency associated to the final selection in the $\gamma \gamma b \bar{b}$ channel. 


\section{The $\tau \tau b \bar{b}$ channel}

This channel is composed by many sub-channels, due the different $\tau$ decays (schematized in figure 6). The analysis targets both the fully hadronic $\tau \tau$ and one hadronic $\tau$ plus a leptonic one final states using three different trigger paths: electron, muon and hadronic taus.

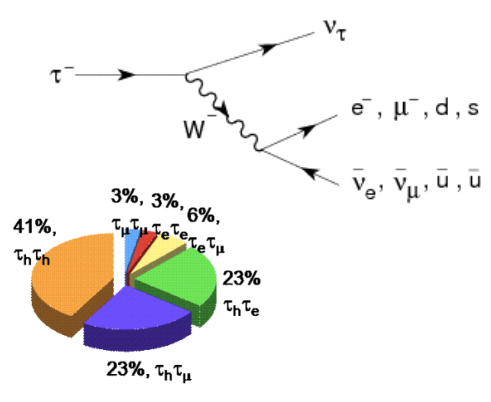

Figure 6: The $\tau$-fermion decays, and branching fractions of a $\tau$-pair.

The hadronic taus are identified by an shower of isolated pions (using $e / \mu$ veto). The full four momenta of the leptonic taus are reconstructed assuming the corresponding neutrinos in collinear approximation. As for the last section two uniquely defined higgs bosons are fully reconstructed. The selected events are categorized as containing two or one medium $b$-tags .

Depending in the $\tau$ decay mode and $b$-tag categorization the dominant background is QCD or $t \bar{t}$, with a non-negligible component of electroweak processes. Figure (7) schematizes the background composition in each category in the di-jet invariant mass after objects pre-selection. The invariant mass of both Higgses candidates are forced to be $125 \mathrm{GeV}$ using a kinematic fit. Events with di-jet mass between $70 \mathrm{GeV}$ and $150 \mathrm{GeV}$ are used for signal extraction. The signal is extracted with a binned maximum likelihood fit in the 4-body mass.

The target of this analysis was to search for SUSY-like signals, the search extends up to 350 $\mathrm{GeV}$ (where the $H \rightarrow h h$ signal is preferred over other signatures in a MSSM low $\tan \beta$ scenario $[8])^{1}$. Figure (8) shows how the final limits for a di-higgs resonance in the $\tau \tau b \bar{b}$ channel splits in the three considered $\tau$-pair decays. Near the signal threshold up to $m_{h h} \sim 320 \mathrm{GeV}$ the cleaner $\tau_{\mu} \tau_{h}$ channel dominate sensibility. For higher $m_{h h}$, where the background statistics drop, the sensibility start to be dominated by the fully hadronic channel.

\footnotetext{
${ }^{1}$ The reference [4] also contains results for the search for a pseudo-scalar boson decaying to a $Z h$ boson pairs, where the Z-boson decay leptonically, what is out of the scope of this contribution.
} 

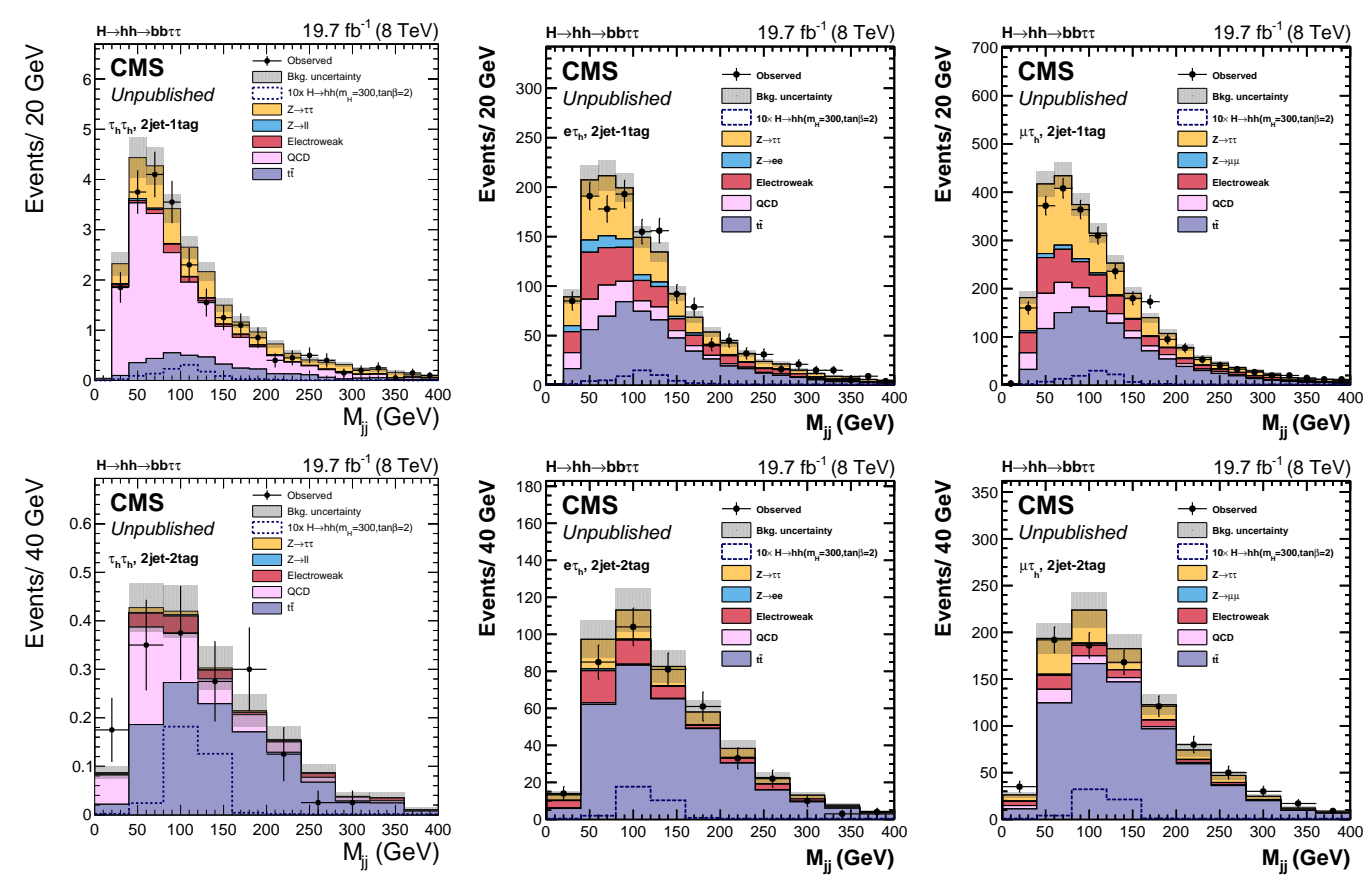

Figure 7: Di-jet invariant mass in the different categories used for the $\tau \tau b \bar{b}$ channel. The first column stands for fully hadronic decays of the $\tau$-pair, while the middle and left columns corresponds to the semi-leptonic decays, respectively in the $e$ and $\mu$ channels. The first row stands for one $b$-tagged jet category, while the second for two $b$-tagged jet category.

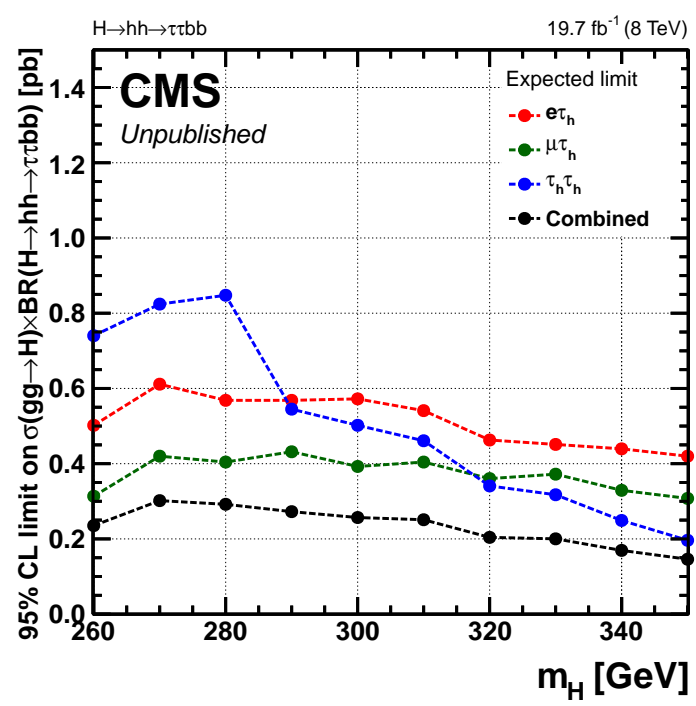

Figure 8: The observed limits of the $\tau \tau b \bar{b}$ channel and its composition in the $\tau$ sub-channels. 


\section{The $b \bar{b} b \bar{b}$ channel}

The events are triggered by the presence of multi-jets, combined with several layers of jet $p_{T}$ pseudo-rapidity. The biggest challenge of the $b \bar{b} b \bar{b}$ channel is the high BKG rate, mainly composed by QCD multijet and $\sim 20 \%$. At least four aKt04 jets and two medium CSV b-tag are required already at trigger level to further reduce QCD rate. The object selection requires 4 central jets $(\eta<$ 2.4) with CMVA b-tagged jets with, with $p_{T}>40 \mathrm{GeV}$. The jets are them paired to create two higgses candidates such that $\left|m_{H}-125 \mathrm{GeV}\right|<35 \mathrm{GeV}$. Selected di-higgs candidates are required to have at least two jets with $p_{T}>90 \mathrm{GeV}$.

Further selections divide the search in Low Mass (LM, when $m_{X}<450 \mathrm{GeV}$ ) and High Mass (HM) region (for $m_{X}>450 \mathrm{GeV}$ ). In the HM region the higgs bosons are supposed to have a considerable boost, therefore an additional selection in the distance of the two jets that compose each higgs candidate $(R<1.5)$ helps to indicate signal. To $m_{h h}>740 \mathrm{GeV}$ there one more additional selection of $p T>300 \mathrm{GeV}$ is added. The signal region is defined selecting the paired di-jet mass around $125 \mathrm{GeV}$, and extracted using a unbined fit in the 4 body mass. The BKG estimation procedure is verified with validation signal and side-bands regions, as schematized in figure (9).

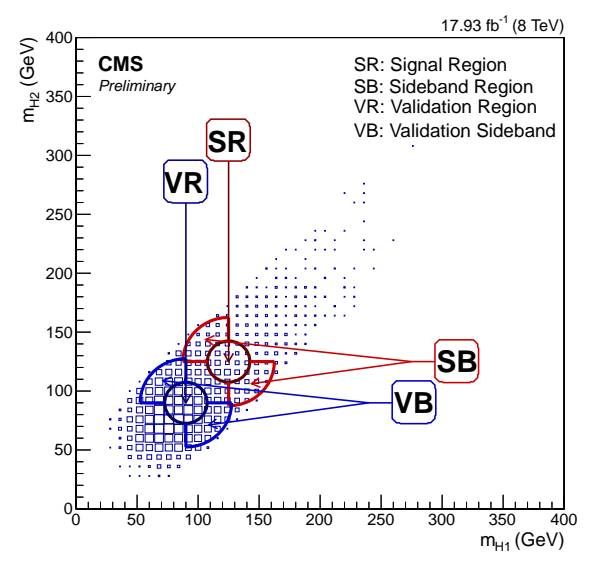

Figure 9: Scatter plot of data in the di-higgs plane, after pre-selection in the $b \bar{b} b \bar{b}$ channel.

The first two captions of figure (10) shows the distribution of data in both HM and LM selections in 4 body mass, as well the signal cut flow efficiency in term of the resonance mass. Background is modeled by a convolution a Gaussian function continued smoothly to exponential tail models the BKG. The normalization is fixed using the data side-bands. The additional selections in $\mathrm{HM}$ region reduces significantly the BKG statistics.

The left side of figure (10) shows the distribution of data in both HM and LM selections in 4 body mass, as well the signal cut flow efficiency in term of the resonance. Around $m_{X} \sim 800 \mathrm{GeV}$ only the object selection start to loose efficiency. This is expected since the search asks for four jets, and around that mass the higgses decays start to merge in one fat jet [9].

Although the search is designed using a narrow scalar signal (the same as described in section 1) the results of this search are also derived for the case of a spin-2 particle, inspired by KKGraviton particles from Warped Extra Dimensional models when the SM fields are allowed to 
propagate in the extra dimensional bulk (see for example [10]). The main differences among the scalar and bulk-like spin-2 case arrives because the angular distribution between the product of the resonance decays (the higgs bosons) are different in the two cases, what influenced the jet combinatorics necessary to identify the higgses. Such a difference is not observed in the $\gamma \gamma b \bar{b}$ channel, since there the higgs bosons are uniquely determined and no angular cut is imposed to them.
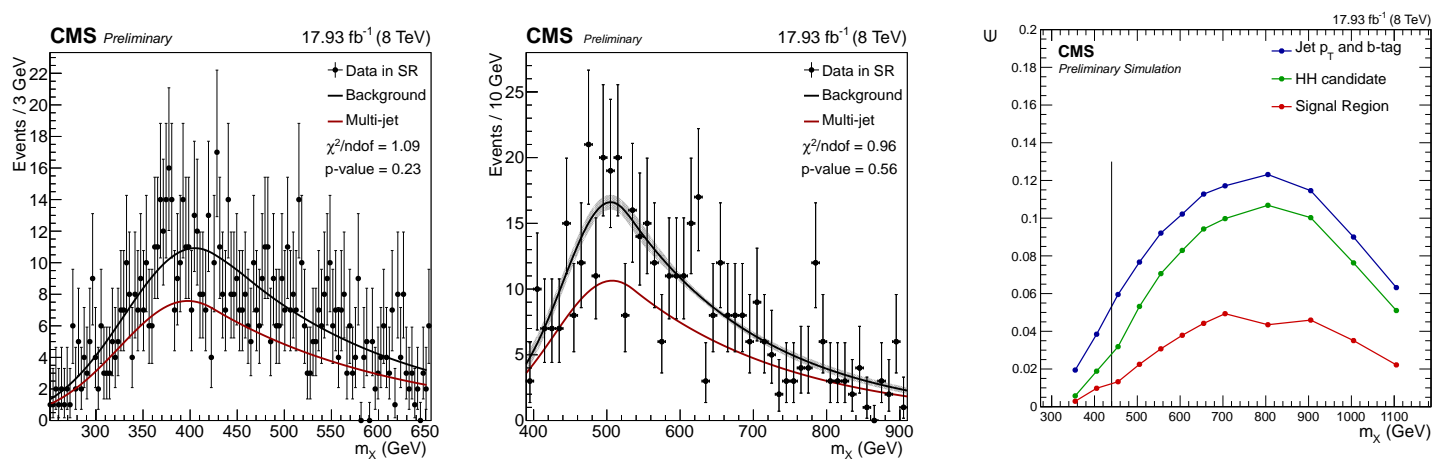

Figure 10: Data distribution in the signal region after LM (left) and HM (middle) selections. The relative contribution of the QCD multijet BKG. The right plot shows the cut flow of signal selection in terms of the resonance mass.

\section{Conclusion}

In this proceeding we had highlighted three CMS searches targeting the di-higgs boson final state. All the Run 1 results up to the moment are performed looking for resonant enhancements. We had focused in the explain that in different mass regimes the different channels (and sub-channels) have different relative contributions, also different mass regimes for the resonance requires adapted analyses strategies. The di-higgs channel is the first channel that can allow measurement of the higgs self-couplings, therefore it is a key channel in the comprehension of the nature of the physics behind the SM formulation. the resonant Run 1 results motivates the future non-resonant searches for BSM phenomena in the higgs boson (self-)couplings to be constructed upon representative signal benchmarks [11] to actually allow detection of higgs boson couplings deviations.

\section{References}

[1] Serguei Chatrchyan et al. "Observation of a new boson at a mass of $125 \mathrm{GeV}$ with the CMS experiment at the LHC”. In: Phys. Lett. B716 (2012), pp. 30-61. DOI: 10.1016 / j • phys letb.2012.08.021. arXiv:1207.7235 [hep-ex].

[2] Georges Aad et al. "Observation of a new particle in the search for the Standard Model Higgs boson with the ATLAS detector at the LHC". In: Phys. Lett. B716 (2012), pp. 1-29. DOI: 10.1016/j.physletb.2012.08.020. arXiv:1207.7214 [hep-ex] .

[3] CMS Collaboration. "Search for the resonant production of two Higgs bosons in the final state with two photons and two bottom quarks". In: (2014). 
[4] CMS Collaboration. "Search for a heavy scalar boson decaying to a pair of $125 \mathrm{GeV}$ Higgs bosons (hh) or for a heavy pseudoscalar boson decaying to $\mathrm{Zh}$, with tau leptons in the final state". In: (2015).

[5] Vardan Khachatryan et al. "Search for resonant pair production of Higgs bosons decaying to two bottom quarkâĂŞantiquark pairs in protonâĂŞproton collisions at $8 \mathrm{TeV}$ ”. In: Phys. Lett. B749 (2015), pp. 560-582. DOI: 10.1016 / j . physletb.2015.08.047. arXiv: 1503.04114 [hep-ex].

[6] Gian F. Giudice, Riccardo Rattazzi, and James D. Wells. "Graviscalars from higher dimensional metrics and curvature Higgs mixing”. In: Nucl. Phys. B595 (2001), pp. 250-276. DOI: 10.1016/S0550-3213(00)00686-6. arXiv:hep-ph/0002178 [hep-ph] .

[7] Vardan Khachatryan et al. "Measurement of Differential Cross Sections for Higgs Boson Production in the Diphoton Decay Channel in pp Collisions at $\sqrt{s}=8 \mathrm{TeV}$ ". In: (2015). arXiv: 1508.07819 [hep-ex].

[8] Abdelhak Djouadi and Jeremie Quevillon. "The MSSM Higgs sector at a high $M_{S U S Y}$ : reopening the low $\tan \beta$ regime and heavy Higgs searches”. In: JHEP 10 (2013), p. 028. DOI: 10.1007 / JHEP10 (2013) 028. arXiv:1304.1787 [hep-ph] .

[9] Maxime Gouzevitch, Alexandra Oliveira, Juan Rojo, Rogerio Rosenfeld, Gavin P. Salam, and Veronica Sanz. "Scale-invariant resonance tagging in multijet events and new physics in Higgs pair production”. In: JHEP 07 (2013), p. 148. DOI: 10 . 1007 / JHEP 07 (2013) 14 8. arXiv:1303.6636 [hep-ph] .

[10] Alexandra Oliveira. "Gravity particles from Warped Extra Dimensions, a review. Part I - KK Graviton". In: (2014). arXiv:1404.0102 [hep-ph] .

[11] Martino Dall'Osso, Tommaso Dorigo, Carlo A. Gottardo, Alexandra Oliveira, Mia Tosi, and Florian Goertz. "Higgs Pair Production: Choosing Benchmarks With Cluster Analysis". In: (2015). arXiv:1507.02245 [hep-ph] . 